\title{
Raman Spectroscopy for the Analysis of Temperature-Dependent Plastic Relaxation of SiGe Layers
}

\author{
F. Pezzoli*, E. Bonera, M. Bollani, S. Sanguinetti, E. Grilli, M. Guzzi \\ L-NESS, Dipartimento di Scienza dei Materiali della Università degli Studi di Milano-Bicocca \\ Via Cozzi 53, I-20125 Milano, Italy \\ G. Isella, D. Chrastina And H. von Känel \\ L-NESS, Dipartimento di Fisica del Politecnico di Milano, Via Anzani 42, I-22100, Como, Italy
}

Novel architectures for electronics and photonics are expected to be developed using the forthcoming $\mathrm{Si}_{1-x} \mathrm{Ge}_{x}$ technology. However, in $\mathrm{Si}_{1-x} \mathrm{Ge}_{x}$-based heterostructures, materials and design issues rely on accurate control of strain and composition of the alloy. The Raman spectroscopy has rapidly emerged as a reliable technique for the quantitative determination of such parameters on a sub-micrometric scale. In this work we present an investigation of the effects of the growth conditions of $\mathrm{Si}_{1-x} \mathrm{Ge}_{x}$ graded layers on dislocation nucleation and interaction. In particular, we focus on the crucial role the deposition temperature plays in the dislocation kinetics. The analysis of threading dislocation densities is accompanied by a quantitative measurement of the residual strain in $\mathrm{Si}_{1-x} \mathrm{Ge}_{x} / \mathrm{Si}$ heterostructures, carried out by means of the Raman scattering. Our approach is effective in studying the physical mechanism governing dislocation multiplication and the sharp transition from a state of brittleness to a state of ductility within a narrow temperature window.

PACS numbers: 61.72.Lk, 61.72.uf, 62.20.mj, 62.20.mm, 78.30.-j

\section{Introduction}

While in the past the striking increase in performance of Si based microelectronic was achieved mainly by reducing the device size, in recent years a new approach has emerged, consisting of the use of materials having optimal characteristics for a given application [1]. In this perspective $\mathrm{Si}_{1-x} \mathrm{Ge}_{x}$ alloys are of particular interest since they are compatible with standard Si CMOS processes: $\mathrm{Si}_{1-x} \mathrm{Ge}_{x}$ has higher bulk mobilities than silicon, and $\mathrm{Si}_{1-x} \mathrm{Ge}_{x} / \mathrm{Si}$ heterostructures allow band gap and strain engineering $[2,3]$. Nevertheless, once a thick $\mathrm{Si}_{1-x} \mathrm{Ge}_{x}$ layer is grown directly on top of a silicon wafer, above a critical thickness, relaxation occurs via the injection into the epilayer of a large number of dislocations, resulting in a threading dislocation density (TDD) larger than what can be tolerated by many electronic and optoelectronic devices [4-6]. As a consequence, there have been several methods developed in order to attempt to obtain high quality $\mathrm{Si}_{1-x} \mathrm{Ge}_{x} / \mathrm{Si}$ buffers, a combination which is usually referred to as a virtual substrate (VS) [7]. One of the most successful approaches in terms of surface

\footnotetext{
* Present address: Institute for Integrative Nanosciences, IFW-Dresden, Helmholtzstr. 20, D-01069 Dresden, Germany. Corresponding author; e-mail: f.pezzoli@ifw-dresden.de
}

morphology and final device performance, is the thick, compositionally graded $\mathrm{Si}_{1-x} \mathrm{Ge}_{x}$ buffer layer [8-11]. In practice, the requirement for low defect densities is met by slowly increasing the Ge content as the thickness of the film increases, from zero to the desired composition. In contrast to constant-composition films, the grading rate offers an additional degree of freedom for controlling the strain at the growth front $[10,12,13]$. Extensive research has been devoted to the optimization of the Ge gradient $[1,11]$. Low TDD, in the $10^{5}-10^{6} \mathrm{~cm}^{-2}$ range, were obtained on buffers with compositional gradients of the order of $10 \% / \mu \mathrm{m}$ or less $[11,14-16]$. Nevertheless, a basic and detailed knowledge of the effects of some growth parameters on dislocation multiplication and interaction is still lacking. While the role of the deposition temperature is expected to be crucial in the determination of the TDD, because it strongly affects dislocation nucleation and kinetics, its effect on TDD multiplication and interaction is not clearly understood. To study this further, for $\mathrm{Si}_{0.6} \mathrm{Ge}_{0.4} / \mathrm{Si}$ graded buffers we correlated Raman measurements of residual strain and TDD data determined by means of optical microscopy and atomic force microscopy (AFM). Our results demonstrate the existence of a sharp increase in the TDD in a narrow window of the deposition temperature. In systems at low plastic strain, such as $\mathrm{Si}_{1-x} \mathrm{Ge}_{x}$ VSs, this rapid and massive dislocation generation can be explained by a brittle 
to ductile transition (BDT). Noticeably, our findings are in good agreement with BDT temperature predicted by a model based on a statistical mechanics description of spontaneous dislocation generation [17].

\section{Experimental details}

A series of $\mathrm{Si}_{0.6} \mathrm{Ge}_{0.4} / \mathrm{Si}(100)$ graded buffers were grown by low energy plasma enhanced chemical vapour deposition (LEPECVD) [18] at a rate of $5-10 \mathrm{~nm} \mathrm{~s} \mathrm{~s}^{-1}$ with the same composition gradient of about $7 \% / \mu \mathrm{m}$, on RCA cleaned 4 inch $\mathrm{Si}(001)$ wafers which had been outgassed for $10 \mathrm{~min}$ at $300^{\circ} \mathrm{C}$. For all the samples the temperature was kept constant at $740^{\circ} \mathrm{C}$, while the $\mathrm{Ge}$ content of the buffer was increased until $x_{\mathrm{Ge}}=0.1 \mathrm{had}$ been reached. At this point the temperature was linearly decreased as the Ge content in the layer was increased. Different temperature vs. Ge content profiles were used such that the final $\mathrm{Si}_{0.6} \mathrm{Ge}_{0.4}$ layer, which was always $1 \mu \mathrm{m}$ thick, was deposited at temperatures ranging between $520^{\circ} \mathrm{C}$ and $580^{\circ} \mathrm{C}$. Diluted Schimmel etch solution was used to reveal TDDs; etch-pit counting was performed by AFM and Nomarski optical microscopy.

In order to probe the top-most epilayer of interest, high-resolution Raman measurements were carried out using the $458 \mathrm{~nm}$ line of an $\mathrm{Ar}^{+}$laser. The scattered light was analysed with a Jasco $800 \mathrm{~mm}$ double grating monochromator and detected with a CCD detector. The spectral pitch of the detector was $0.3 \mathrm{~cm}^{-1}$. Finally, the laser power was chosen in order to limit the sample heating shifts to well below the measurements noise, and the measured Raman spectra were calibrated by simultaneously collecting the emission lines of spectral lamps.

\section{Discussion}

It has been suggested that composition, $x$, and biaxial strain, $\epsilon$, of $\mathrm{Si}_{1-x} \mathrm{Ge}_{x} / \mathrm{Si}$ heterostructures can be measured directly from one Raman spectrum only [19]. However, it is worth noting that the composition effect, which determines the three mode peak positions, is superimposed on the effect of the macroscopic biaxial strain. In these epitaxial systems the analysis of the Raman spectra is thus not straightforward and a precise measurement of strain and composition can be performed only if relations describing the $x$ and $\epsilon$ dependence of the $\mathrm{Si}-\mathrm{Si}, \mathrm{Si}-\mathrm{Ge}$ and $\mathrm{Ge}-\mathrm{Ge}$ Raman active phonon modes are well known. Recently we compared Raman measurements of $\mathrm{Si}_{1-x} \mathrm{Ge}_{x} / \mathrm{Si}$ heterostructures with high resolution X-ray diffraction data. The results, outlined in Ref. [20], demonstrate that the following dependencies:

$$
\begin{aligned}
& \omega^{\mathrm{Si}-\mathrm{Si}}(x, \epsilon)=520.7-66.9 x-730 \epsilon, \\
& \omega^{\mathrm{Si}-\mathrm{Ge}}(x, \epsilon)=400.1+24.5 x-4.5 x^{2}-33.5 x^{3} \\
& \quad-570 \epsilon, \\
& \omega^{\mathrm{Ge}-\mathrm{Ge}}(x, \epsilon)=280.3+19.4 x-450 \epsilon
\end{aligned}
$$

are reliable and effective for the quantitative determination of strain and composition of $\mathrm{Si}_{1-x} \mathrm{Ge}_{x} / \mathrm{Si}$ het- erostructures, enabling the exploitation of fundamental properties of such systems. Hence, here we evaluated $x$ and $\epsilon$ of the samples studied in this work, using Eqs. (1)-(3) and employing the procedure described in detail in Ref. [20]. Raman analysis provided the alloy composition for all the samples, showing that it is the same, within the experimental uncertainties (Fig. 1). Raman results summarized in Fig. 2 demonstrated also that the samples can be considered relaxed, as shown in similar samples [21], because of their low strain values of the order of some $10^{-4}$.

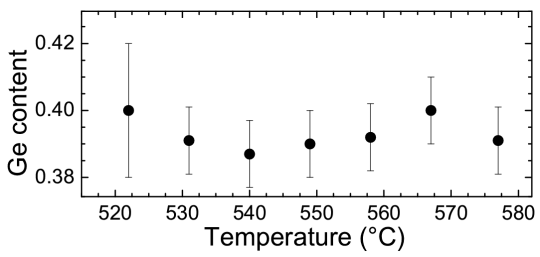

Fig. 1. Ge content of the alloy, obtained by Raman analysis, as a function of the deposition temperature.

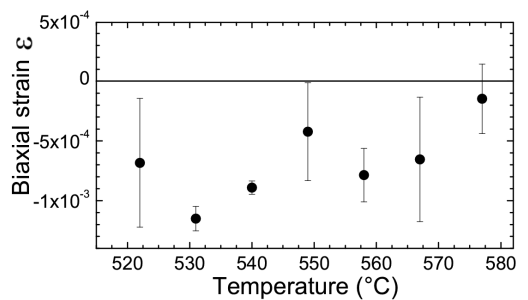

Fig. 2. Biaxial strain obtained by Raman analysis as a function of the deposition temperature.

TABLE

Growth temperature and TDD obtained by AFM (A) and Nomarski (B) measurements of the studied samples. RMS roughness, measured by AFM, is also summarized.

\begin{tabular}{c|c|c|c}
\hline \hline$T\left[{ }^{\circ} \mathrm{C}\right]$ & TDD-A $\left[\mathrm{cm}^{-2}\right]$ & TDD-B $\left[\mathrm{cm}^{-2}\right]$ & RMS $[\mathrm{nm}]$ \\
\hline 522 & $1.0 \times 10^{5}$ & $1.4 \times 10^{5}$ & 3.0 \\
531 & $1.5 \times 10^{5}$ & $2.0 \times 10^{5}$ & 2.9 \\
540 & $2.0 \times 10^{5}$ & $3.1 \times 10^{5}$ & 2.9 \\
549 & $1.4 \times 10^{6}$ & $1.1 \times 10^{6}$ & 3.0 \\
558 & $1.7 \times 10^{6}$ & $1.9 \times 10^{6}$ & 2.7 \\
567 & $2.5 \times 10^{6}$ & $1.9 \times 10^{6}$ & 2.8 \\
577 & $2.4 \times 10^{6}$ & $1.9 \times 10^{6}$ & 3.0
\end{tabular}

As is clearly visible from Table the TDD suddenly increases from $2 \times 10^{5} \mathrm{~cm}^{-2}$ to $2 \times 10^{6} \mathrm{~cm}^{-2}$, when the graded buffer is deposited following a temperature profile corresponding to a final temperature of $540^{\circ} \mathrm{C}$. The smooth decrease in the biaxial strain with the increase of the deposition temperature from about $1 \times 10^{-3}$ at $T \approx 530^{\circ} \mathrm{C}$ to nearly complete relaxation at $580^{\circ} \mathrm{C}$ (see 
Fig. 2) cannot, however, justify the increase of about one order of magnitude in the TDD. A reasonable explanation of such behavior is based on a BDT. The BDT has been known for a long time to be related to an increase in the dislocation density, but this mechanism has never been studied in heteroepitaxial systems. The significant point about the data reported in Table is the massive increase in the TDD at a critical temperature of $540^{\circ} \mathrm{C}$ and the near coincidence of this point with the BDT temperature of the alloy $\left(\leq 600^{\circ} \mathrm{C}\right)$ given by the Khantha model [17, 22]. Remarkably, our findings are in agreement with previous reports on $\mathrm{Si}$, i.e. that the transition range is only a few degrees $\left(\leq 10^{\circ} \mathrm{C}\right)[23,24]$ and the observed transition temperature is about half the alloy melting point $[17,25]$. Finally, the thermal stability of the samples has been confirmed by an additional sample deposited with a final temperature of $520^{\circ} \mathrm{C}$ and annealed for $15 \mathrm{~min}$ at $740^{\circ} \mathrm{C}$. In this heterostructure we observed an increase in the degree of relaxation and no significant variation in the TDD as compared to the as grown sample.

\section{Summary and conclusions}

The strain-relieving mechanisms in $\mathrm{Si}_{1-x} \mathrm{Ge}_{x} / \mathrm{Si}$ heterostructures pose a number of basic questions concerning the role of growth parameters and their consequences on defect dynamics. It is clear that the understanding of the vibrational properties is increasingly important because it provides the means with which one can study these phenomena. The main aim of this work was thus to study the lattice dynamics of $\mathrm{Si}_{0.6} \mathrm{Ge}_{0.4} / \mathrm{Si}$ graded buffers in order to investigate the role of dislocations on the mechanical properties of the alloy. The growth of thick graded buffers, studied in this work, is demanding for conventional deposition techniques such as MBE, mainly because of the very high growth rates required to obtain a shallow Ge gradient profile within a reasonable process time. Furthermore, the massive increase in the TDD, within less than $10^{\circ} \mathrm{C}$, was observed in a low deposition temperature window, not accessible with common CVD. The main features of LEPECVD, namely a high growth rate at a low substrate temperature, have indeed been employed to gather insight into defect nucleation in $\mathrm{Si}_{1-x} \mathrm{Ge}_{x} / \mathrm{Si}$ heterostructures. We have shown that a sharp increase in the TDD takes place at a critical temperature in good agreement with the brittle to ductile transition temperature predicted by a literature model.

\section{Acknowledgments}

This study was supported by the Cariplo Foundation, SIMBAD and MANDIS projects.

\section{References}

[1] F. Schäffler, Semicond. Sci. Technol. 12, 1515 (1997).

[2] F. Capasso, Science 235, 172 (1987).
[3] D.J. Paul, Semicond. Sci. Technol. 19, R75 (2004).

[4] G. Schuberth, F. Schäffler, M. Besson, G. Abstreiter, E. Gornik, Appl. Phys. Lett. 59, 3318 (1991).

[5] K. Ismail, B.S. Meyerson, P.J. Wang, Appl. Phys. Lett. 58, 2117 (1991).

[6] K. Ismail, J. Vac. Sci. Technol. B 14, 2776 (1996).

[7] E. Kasper, K. Lyutovich, M. Bauer, M. Oehme, Thin Solid Films 336, 319 (1998).

[8] F.K. LeGoues, B.S. Meyerson, J.F. Morar, Phys. Rev. Lett. 66, 2903 (1991).

[9] M.S. Abrahams, L.R. Weisberg, C.J. Buiocchi, J. Blanc, J. Mater. Sci. 4, 223 (1969).

[10] J. Tersoff, Appl. Phys. Lett. 62, 693 (1993); erratum ibid. 64, 2748 (1994).

[11] E.A. Fitzgerald, Y.-H. Xie, M.L. Green, D. Brasen, A.R. Kortan, J. Michel, Y.-J. Mii, B.E. Weir, Appl. Phys. Lett. 59, 811 (1991).

[12] E. Koppensteiner, P. Hamberger, G. Bauer, V. Holý, E. Kasper, Appl. Phys. Lett. 64, 172 (1994).

[13] P.M. Mooney, J.L. Jordan-Sweet, J.O. Chu, F.K. LeGoues, Appl. Phys. Lett. 66, 3642 (1995).

[14] M. Hohnisch, H.J. Herzog, F. Schäffler, J. Crystal Growth 157, 126 (1995).

[15] E.A. Fitzgerald, Y.-H. Xie, D. Monroe, P.J. Silverman, J.M. Kuo, A.R. Kortan, F.A. Thiel, B.E. Weir, J. Vac. Sci. Technol. B 10, 1807 (1992).

[16] S. Marchionna, A. Virtuani, M. Acciarri, G. Isella, H. von Känel, Mater. Sci. Semicond. Process. 9, 802 (2006).

[17] M. Khantha, D.P. Pope, V. Vitek, Phys. Rev. Lett. 73, 684 (1994).

[18] C. Rosenblad, H.R. Deller, A. Dommann, T. Meyer, P. Schroeter, H. von Känel, J. Vac. Sci. Technol. A 16, 2785 (1998).

[19] J.C. Tsang, P.M. Mooney, F. Dacol, J.O. Chu, J. Appl. Phys. 75, 8098 (1994).

[20] F. Pezzoli, E. Bonera, E. Grilli, M. Guzzi, S. Sanguinetti, D. Chrastina, G. Isella, H. von Känel, E. Wintersberger, J. Stangl, G. Bauer, to be published in Mat. Sci. Semicond. Process., doi:10.1016/j.mssp.2008.09.012 .

[21] F. Pezzoli, Lucio Martinelli, E. Grilli, M. Guzzi, S. Sanguinetti, M. Bollani, D. Chrastina, G. Isella, H. von Känel, E. Wintersberger, J. Stangl, G. Bauer, Mater. Sci. Eng. B 124125, 127 (2005).

[22] M. Khantha, D.P. Pope, V. Vitek, Mater. Sci. Eng. A 192-193, 435 (1995).

[23] P.B. Hirsch, S.G. Roberts, Philos. Mag. A 64, 55 (1991).

[24] P. Pirouz, A.V. Samant, M.H. Hong, A. Moulin, L.P. Kubin, J. Mater. Res. 14, 2783 (1999).

[25] H.J. Herzog, in: Properties of Silicon Germanium and SiGe:Carbon, Eds. E. Kasper, K. Lyutovich, number 24, EMIS Datareviews, 1999, p. 45. 